\title{
Symmetry Fundamentalism: A Case Study from Classical Physics
}

\author{
David Schroeren \\ Princeton University* \\ May 28, 2020 \\ Forthcoming in The Philosophical Quarterly
}

\begin{abstract}
Physicists have suggested what I call symmetry fundamentalism: the view that symmetries are fundamental aspects of physical reality and that these aspects are more fundamental than what one might ordinarily think of as the fundamental building blocks of the world, such as elementary particles. The goal of this paper is to develop an ontology for classical particle mechanics that provides a precise instance of symmetry fundamentalism.
\end{abstract}

\section{Introduction}

In the second half of the 20th century, fundamental physics experienced a major breakthrough: physicists noticed the incredible power of the notion of symmetry. Just on the basis of claims about symmetries, physicists were able to infer claims about the nature of physical properties as well as about the nature and behaviour of matter - claims that were of unprecedented empirical accuracy.

This paradigm shift towards symmetries gives rise to an intriguing metaphysical vision. When an entity plays a central role in the inferential practice of physics, we are used to entertaining the hypothesis that this entity is metaphysically fundamental. The truth of such a hypothesis would be a particularly compelling vindication of this practice: the relevant entities are inferentially significant precisely because of their metaphysical fundamentality. In this vein, physicists have suggested what I refer to as symmetry fundamentalism: the view that symmetries are fundamental aspects of physical reality, whereas the physical entities that we would ordinarily have thought of as the fundamental building blocks of the physical world - such as elementary particles or fields - are ontologically derivative of these aspects. For example, Steven Weinberg has argued that "symmetries are fundamental" 1 and thus that "at the deepest level,

\footnotetext{
*The Department of Philosophy, 1879 Hall, Princeton University, Princeton, NJ 08544, United States. E-mail: schroeren@princeton.edu.

${ }^{1}$ (Weinberg, 1987, p. 79).
} 
all we find are symmetries and responses to symmetries," ${ }^{2}$ whereas "matter [...] loses its central role in physics." ${ }^{3}$ According to Werner Heisenberg, we should "replace the concept of a particle" with "the concept of a fundamental symmetry", and "what we have to look for are not fundamental particles but fundamental symmetries." 4

There are serious obstacles to making sense of this view. Symmetries are part of the mathematical apparatus used to describe the physical world. But symmetry fundamentalism is not the idea that physical reality is literally made of mathematical objects like sets and functions. It is a thesis about the physical constituents of fundamental reality. To give precise content to symmetry fundamentalism, we therefore need to articulate an ontology which identifies the physical entities (such as objects, properties and relations) that correspond to mathematical symmetry transformations and which makes transparent how particles and fields can be regarded as ontologically derivative of these entities. ${ }^{5}$ The goal of this paper is to do just that.

I will focus on the example of classical particle mechanics: the theory whose standard ontology consists of point particles evolving according to Newton's equations of motion. Call this the individualistic ontology. Taking inspiration from the symmetry structure of the theory, I articulate an alternative ontology that vindicates symmetry fundamentalism. But this paper is not primarily intended as a contribution to the metaphysics of classical mechanics. Rather, the case study illustrates symmetry-fundamentalist ontological theorizing by appeal to a familiar example and serves as a guide for the development of corresponding ontological proposals for quantum theory - a project I carry out in another paper. ${ }^{6}$

My goal in this paper is not to give a systematic defense of symmetry fundamentalism, but rather to develop a precise account of what it would be for symmetry fundamentalism to be true at a world consisting of classical point particles. In other words: the aim is to detail a classical-mechanical world whose constituent particles are ontologically derivative on the fundamental ontological counterparts of symmetries. This is a substantive challenge: when we are told that entities of one kind are ontologically derivative of entities of another kind, we expect an account that details how every relevant proposition about the former is entailed by some proposition about the latter. One of the main tasks of this paper is therefore to show that, for every proposition about particles statable in the individualistic theory, there is some fundamental proposition about the physical counterparts of symmetries that entails it. Indeed, I will show something even stronger: that every proposition about particles statable in the individualistic theory is equivalent to some fundamental proposition.

\footnotetext{
${ }^{2}$ (Weinberg, 1987, p. 80).

${ }^{3}$ (Weinberg, 1992, pp. 138-9). Steven Weinberg has confirmed in personal correspondence that these remarks were intended as claims about fundamental physical reality, rather than merely about the epistemic or pragmatic significance of symmetry techniques.

${ }^{4}$ (Heisenberg, 1973, p. 273). Cf. Heisenberg (1976, p. 924) and Ne'eman and Sternberg (1991, p. 327)

${ }^{5}$ For the purposes of this paper, an 'ontology' is a hypothesis about the physical constituents (such as objects, properties and relations) of fundamental physical reality according to some physical theory. Moreover, I take it for granted in this paper that entities of any ontological type - such as objects, properties, relations - can be fundamental.

${ }^{6}$ (Schroeren, 2020).
} 
The centrality of symmetries in modern physics has also served as a key inspiration for ontic structural realism, an influential intellectual tradition in the philosophy of science according to which physical reality is fundamentally 'structural' in a way that is to be explicated at least in part by appeal to symmetries. ${ }^{7} \mathrm{~A}$ discussion that does justice to the rich and vibrant structuralist literature on the topic is beyond the scope of this paper. For present purposes, I want to point out two things. First of all, the approach I will pursue does not respect the constraint, expressed by some ontic structuralists as well as other metaphysicians, that an account of fundamental physical reality must not involve first-order individuals: the kind of entity that individual constants refer to or that first-order quantifiers range over. ${ }^{8}$ Second, the ontic structuralist project has been quite controversial: for example, a common complaint is that the operative notion of 'structure' has not been satisfactorily clarified. ${ }^{9}$ Although I am in agreement with ontic structural realists about the metaphysical importance of symmetries in physics, in this paper I will explore whether symmetry fundamentalism can be put on a sound ontological footing without committing to controversial structural realist assumptions. This is a project that should be of interest even to those who favour an ontic structuralist approach.

Here is the plan. Section 2 introduces the notion of symmetry that is involved in symmetry fundamentalism, explains why classical particle mechanics is well-suited as a testbed for symmetry-fundamentalist ontological theorizing and highlights a key advantage of the symmetry-fundamentalist approach. Sections 3 and 4 develop a symmetry-fundamentalist ontology for classical particle mechanics. I conclude in section 5.

\section{State-Space Symmetries}

Symmetry is a notion of which we have an intuitive grasp. A round dinner plate is symmetric under rotations: when I rotate the plate about a specific axis, it looks the same as before. The basic idea is that a symmetry of an object corresponds to ways of changing the object that preserve certain salient features of that object. This basic idea is also reflected in the physical notion of symmetry, albeit in a more abstract form: here, symmetries are mathematical functions (also known as transformations) that preserve certain important features of the mathematical description of the world.

Here is an example. We are familiar with the idea that Euclidean space has symmetries, such as rigid spatial shifts and rigid rotations. These transformations count as symmetries because they preserve what one might think of as the characteristic structural feature of Euclidean space: the Euclidean metric. Symmetries of Euclidean space are transformations that preserve this structure, also known as Euclidean isometries.

This example illustrates something important: abstractly, a symmetry of a structured mathematical space is always defined as a transformation of that space which preserves

\footnotetext{
${ }^{7}$ (Ladyman, 1998; Castellani, 1998; Brading and Castellani, 2003; Kantorovich, 2003; Ladyman et al., 2007; Roberts, 2011; French, 2014; McKenzie, 2014; Brading et al., 2017).

${ }^{8}$ (French and Ladyman, 2003; Ladyman et al., 2007; French, 2010). Other metaphysicians who have developed proposals for dispensing with first-order individuals include Shamik Dasgupta (2009) and Andrew Bacon (2019).

${ }^{9}$ For example, (Dorr, 2010; Wolff, 2011; Arenhart and Bueno, 2015); cf. (McKenzie, 2017).
} 
the characteristic structure of that space. ${ }^{10}$ The symmetries that are involved in symmetry fundamentalism are symmetries in this more abstract sense: they are symmetries of whatever mathematical space corresponds to the state space of the world according to the theory in question. Symmetries of this sort are known as state-space symmetries. ${ }^{11}$

Before I explain why state-space symmetry is the notion of symmetry operative in symmetry fundamentalism, let me tell you what a state-space symmetry is. First of all, a proposition is the instantaneous state of a world at a time iff it is a maximally specific and exhaustive description of the values of the variable fundamental physical quantities at that time: that is, the physical quantities whose values possibly vary between instants of time and whose values at some instant of time, taken together, determine the values of all other physical quantities at that instant. The fact that states are exhaustive and maximally specific entails that states are incompatible: the world cannot be in more than one state at any given instant of time. However, the world is necessarily in some state at every instant of time. States therefore have some similarities to possible worlds. ${ }^{12}$

For example, consider classical (Newtonian) $N$-particle mechanics: the theory that describes the temporal evolution of $N$ point particles in three-dimensional Euclidean space according to Newton's laws of motion. The default ontology for this theory is what I call individualism: the view that fundamental reality consists in point particles instantiating the values of certain fundamental quantities. A common choice for these quantities is position and momentum. Figure 1 is a schematic illustration of a world with two particles.

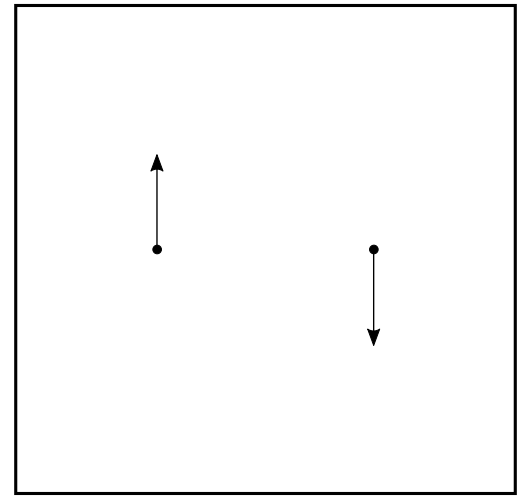

Figure 1: Schematic illustration of a two-particle world at some instant of time.

Call a proposition the complete individualistic profile of a classical particle world at some instant of time iff it fixes the positions and momenta of all particles at that time.

\footnotetext{
${ }^{10}$ In this way, we can think of structured mathematical spaces as individuated by their symmetries along the lines of the Erlanger Programm (Klein, 1893, 1910); cf. (Wallace, 2019) for a more recent statement of this idea.

${ }^{11}$ The notion of a state-space symmetry (also known as a 'Wigner symmetry') is well-entrenched in physics (Wigner, 1931, 1968; Strocchi, 2005; Moretti, 2013). State-space symmetries have also been recognized as a distinctive kind of symmetry in the philosophical literature; e.g. (Earman, 2003, p. 341) and (Kochen, 1996, p. 242).

${ }^{12}$ For more on the connection between states and possible worlds, see (Williamson, 2016).
} 
The complete individualistic profile at some time entails the values of all other physical quantities at that time. According to individualism, instantaneous states are complete individualistic profiles. (I will later propose an ontology for classical particle mechanics according to which instantaneous states and individualistic profiles come apart.)

State space is just the space of all instantaneous states of the world: in the case of classical particle mechanics, the space of all possible combinations of positions and momenta of every particle. In this setting, the mathematical spaces used to characterize state spaces are called phase spaces, illustrated in figure $2 .{ }^{13}$

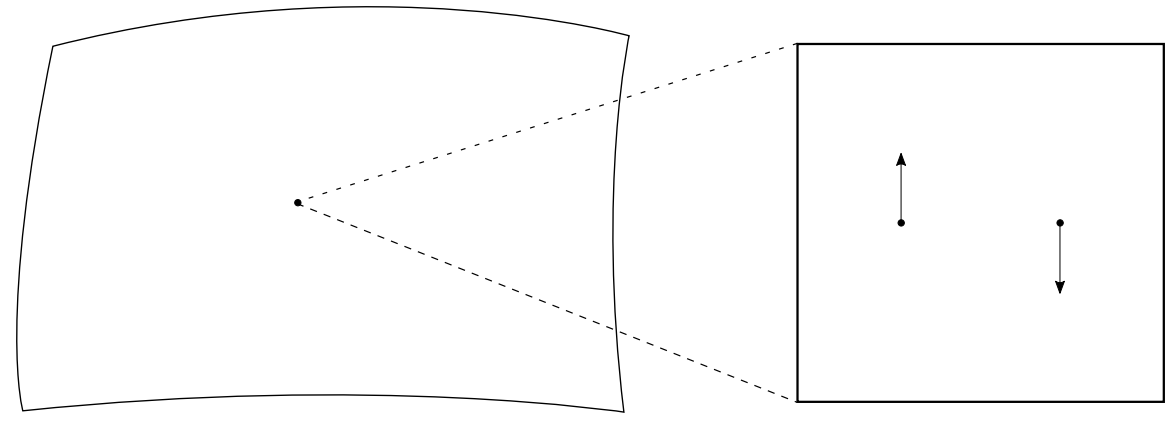

Figure 2: Each point in phase space (shown on the left) corresponds to a situation as shown on the right.

This brings us to the notion of a state space symmetry. Just as a transformation of Euclidean space is a symmetry of Euclidean space iff it preserves the characteristic structural feature of Euclidean space - the Euclidean metric - a transformation of phase space is a symmetry of phase space iff it preserves the characteristic structural feature of phase space: the symplectic form. We won't need the technical definition of this notion in what follows; ${ }^{14}$ to a first approximation, you can think of the symplectic form as a mathematical object that determines the volumes of phase space regions, and of phase space symmetries as transformations that preserve the volumes of such regions. ${ }^{15}$ This is illustrated in figure 3. An example of state-space symmetry are shifts of individual particles in position and momentum, as illustrated in figure 4.

The notion of a state-space symmetry is distinct from the notion of a dynamical symmetry. For present purposes, think of the dynamical law of a classical particle world as a fact about which curves in state space count as the dynamically allowed histories of this world. Mathematically, the dynamically allowed histories correspond to what's called a flow on phase space: a family of phase space curves such that every phase space point

\footnotetext{
${ }^{13}$ The present case study is limited to classical worlds whose state spaces can be described in terms of even-dimensional phase spaces. This assumption can be relaxed; an example is the formalism known as 'Nambu mechanics' (Nambu, 1973).

${ }^{14}$ In technical terms, a symplectic form on a smooth manifold $M$ is a closed non-degenerate differential 2 -form on $M$. Roughly, a differential $n$-form on $M$ is a map from $n$-tuples of smooth vector fields on $M$ to smooth functions on $M$. A differential 2-form $\omega$ on $M$ is closed iff its exterior derivative vanishes; $d \omega=0 . \omega$ is non-degenerate iff the existence of an $X$ such that $\omega(X, Y)=0$ for all $Y$ implies $X=0$.

${ }^{15}$ The reason why this is only a first approximation is that, although every transformation that preserves the symplectic form also preserves the volumes of phase space regions, the converse is not true.
} 


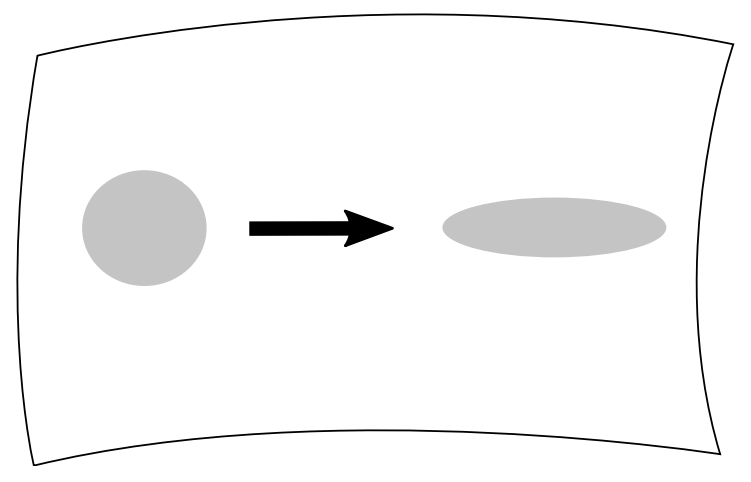

Figure 3: The map that corresponds to the black arrow is a symmetry of phase space only if the two shaded regions are of equal volume.

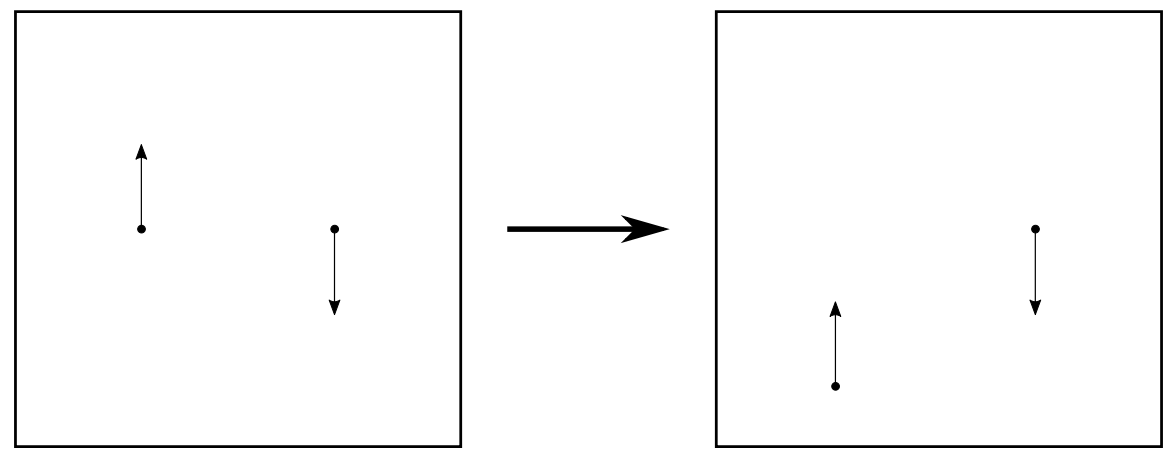

Figure 4: Illustration of the effect of a spatial shift symmetry on a particular state.

is intersected exactly once by exactly one curve in this family. A state-space symmetry is a dynamical symmetry iff it maps dynamically allowed flow lines to other dynamically allowed flow lines. ${ }^{16}$ But although every dynamical symmetry is a state-space symmetry, the converse is not true. ${ }^{17,18}$

I should therefore explain why it is the notion of a state-space symmetry that is operative in symmetry fundamentalism, rather than the notion of a dynamical symmetry.

\footnotetext{
${ }^{16}$ More precisely: a phase space symmetry $T$ is a dynamical symmetry iff for every dynamically allowed flow line $h$, there is another dynamically allowed flow line $h^{\prime}$ such that every phase space point on $h$ gets mapped by $T$ to a unique phase space point on $h^{\prime}$.

${ }^{17}$ An example of a state-space symmetry that isn't a dynamical symmetry are momentum shifts of individual particles. Suppose that the Hamiltonian of the two-particle world is that of a free system: $H=\frac{1}{2 m}\left(p_{A}^{2}+p_{B}^{2}\right)$, where $p_{A}$ and $p_{B}$ denote the momentum coordinate functions of particles $A$ and $B$ respectively. Shifts in the momentum of particle $A$ are generated by its position coordinate function $q_{A}$. Moreover, shifts in the momentum coordinate of $A$ are a dynamical symmetry only if $\left\{q_{A}, H\right\}=0$. But $\left\{q_{A}, H\right\}=\frac{p_{A}}{m}$, so momentum shifts of $A$ are not a dynamical symmetry.

${ }^{18}$ Note: the fact that not every state-space symmetry is a dynamical symmetry entails that not every state-space symmetry satisfies the constraint that symmetry-related states are indistinguishable from one another; cf. (Dasgupta, 2016). Intuitively: there are lots of ways of shifting only one particle in physical space that do not result in an indistinguishable situation.
} 
The main reason is that, whether a given state-space symmetry is also a dynamical symmetry plays no role in the relevant symmetry-based inferences; and if a dynamical symmetry figures in such an inference, it does so only in virtue of the fact that it is a state-space symmetry. Perhaps the best illustrations of this can be found in quantum theory, where state space is characterized by Hilbert space: a vector space endowed with an inner product, a function you can roughly think of as an assignment of lengths to vectors. In this context, state-space symmetries are Hilbert space transformations that preserve the inner product. ${ }^{19} \mathrm{~A}$ full discussion of the quantum case is beyond the scope of this paper. ${ }^{20}$ Here, I'll just note two important examples. First, the only symmetryrelated premise in the characterization of spin in non-relativistic quantum mechanics is that rotations are state-space symmetries. ${ }^{21}$ Similarly, the only symmetry-related premise in the classification of elementary particle kinds in terms of mass and spin is that relativistic boosts, rotations, and shifts (also known as Poincaré transformations) are state-space symmetries. ${ }^{22}$ Both are paradigmatic examples of symmetry-based inferences that motivate the symmetry-fundamentalist vision expressed by Weinberg and others; but neither requires an assumption about whether the state-space symmetries in question also count as dynamical symmetries.

In this context, it is important to point out why it might be tempting to conflate state-space symmetries with dynamical symmetries: standard introductions of symmetry techniques in physics often focus on physical systems whose dynamical and state-space symmetries coincide. ${ }^{23}$ An example of such a system is a classical Newtonian particle world that contains exactly one particle in three-dimensional Euclidean space and whose dynamics are governed by a Hamiltonian of the form $H=\frac{p^{2}}{2 m}+V(x)$, where $V(x)$ is a spherically symmetric potential. In this world, rotations are symmetries of both kinds: they are state-space symmetries (since they preserve the symplectic form); and they are dynamical symmetries (because they leave $H$ invariant). But this coincidence breaks down in worlds that contain multiple particles. In such a world, rotations of an individual particle count as state-space symmetries but generally do not count as dynamical symmetries: for example, any multi-particle Hamiltonian whose potential term depends on particle distances is not invariant under such transformations. ${ }^{24}$

We are now in a position to understand why the classical setting is adequate as a testbed for symmetry-fundamentalist ontological theorizing. State-space symmetries are central to virtually every theory of fundamental physics developed since the time of Heisenberg and Schrödinger, including quantum field theory - the theoretical framework that inaugurated the paradigm shift towards symmetry techniques and that underlies the wildly successful 'standard model of particle physics' - and influential proposals for

\footnotetext{
${ }^{19}$ Hilbert space transformations that preserve the inner product are known as unitary and anti-unitary transformations; cf. (Wigner, 1931; Weinberg, 1995).

${ }^{20}$ A symmetry-fundamentalist ontology for quantum mechanics is developed in (Schroeren, 2020).

${ }^{21}$ For example, the fact that spin is discrete follows from the Peter-Weyl theorem on the assumption that rotations are state-space symmetries (i.e. unitary transformations); this is the only symmetry-related assumption required by the theorem (Folland, 2015, p. 136).

${ }^{22}$ This classification is due to Eugene Wigner (1939).

${ }^{23}$ For example, (Ballentine, 2000, pp. 77).

${ }^{24}$ Both electrostatic and Newtonian gravitational potentials are of this form.
} 
a quantum theory of gravity. ${ }^{25}$ For example, as can be confirmed by consulting standard textbooks on the subject, virtually any transformation that is called a 'symmetry' in quantum field theory is at least a state space symmetry. ${ }^{26}$ Indeed, the continuing and thoroughgoing utility of symmetry techniques in modern physics is likely what inspired physicists like Weinberg to suggest that symmetries should be regarded as corresponding to fundamental aspects of reality. Now, state-space symmetries aren't just an important part of modern quantum physics, but also play a crucial role in the seemingly antiquated setting of classical particle mechanics - specifically, in its Hamiltonian formulation. This means that we find ourselves in the rather fortunate situation of being able to make progress on the ontology of a wide range of cutting-edge physical theories without getting sidetracked by their mathematical complexities. We can use classical particle mechanics as a testbed for symmetry-fundamentalist ontological theorizing without loss of generality.

This highlights a key strength of my approach. Although the predictions of current fundamental physics are more accurate than ever before, there is good reason to think that none of our current best theories provide a true and exhaustive description of fundamental physical reality. For ontological theorizing about existing physics to give us insight into the fundamental structure of the actual world (rather than some possible world in which existing physics is exactly true), we should have good reason to think that the results of our theorizing are likely to apply to more exact and complete future physical theories, or at least that they allow us to discern general strategies for developing an ontology for such theories. This is precisely what seems to be the case for the approach in this paper: since state-space symmetries have been and continue to be at the heart of a wide range of proposals for fundamental physics, the symmetry-fundamentalist approach has a good claim to be capable of delivering candidate ontologies for future fundamental physics as well.

In this section, we've familiarized ourselves with the notion of a state-space symmetry. In the remainder of the paper, I will develop an ontology according to which the physical counterparts of state-space symmetries are fundamental and which details how every relevant proposition about particles can be derived from some fundamental proposition.

\section{State-Space-First Metaphysics}

For individualists, state space is nothing over and above complete individualistic profiles: for the world to be in some state at some instant of time just is for some complete individualistic profile to be true.

But there is an alternative view of state space, one that is key to the symmetryfundamentalist ontology I'm about to develop. According to this state-space-first view,

\footnotetext{
${ }^{25}$ One important example is loop quantum gravity, where the fundamental physical entities are mathematically characterized in terms of spin networks: graphs whose links are each endowed with a Hilbert space that is acted upon by a unitary representation of the rotation group $S U(2)$-i.e. by a group acting in terms of state-space symmetries. Moreover, the fundamental physical quantities (area and volume) are defined in terms of the generators of those state-space symmetries; (Rovelli, 2011).

${ }^{26}$ For example, (Weinberg, 1995, p. 51).
} 
there are fundamental entities (called primitive states) and some appropriate fundamental notion of 'actuality' which satisfies the constraint that, necessarily, for every instant of time there is exactly one primitive state that is actual at that instant. Primitive states have the structure of the individualistic state space and are in one-to-one correspondence with complete individualistic profiles. Although the state-space-first metaphysician agrees with the individualist about which complete individualistic profile is true at any given instant of time, they deny that primitive states are complete individualistic profiles: on the state-space-first view, particles are ontologically derivative and so do not figure in the bottom-line story of physical reality. Primitive states are therefore quite different from their individualistic counterparts: as opposed to individualistic states, when two primitive states are distinct, this is not so by virtue of disagreeing about any particular particle; they are primitively distinct. ${ }^{27}$ This means that, on the state-space-first view, the constraint that no more than one state can be actual at any given instant of time is quite different in nature than it is on the individualistic view. On the individualistic picture, the fact that at most one complete individualistic profile can be true at any given instant of time is a logical consequence of the fact that individualistic profiles are maximally specific and exhaustive, and therefore mutually exclusive. Since the state-space-first metaphysician regards states as primitive entities that are distinct from complete individualistic profiles, they must implement this constraint as a basic law governing the actuality relation.

Since the state-space-first view entails that particles are ontologically derivative, the main challenge for this view is to show that every complete individualistic profile is entailed by some fundamental proposition about primitive states.

It is clear that the requisite fundamental propositions cannot just say which primitive states are actual. Recall: according to the individualist, the proposition that some individualistic state is actual at some instant of time determines the complete individualistic profile at that instant. Since primitive states do not have individualistic structure, the state-space-first metaphysician must deny the corresponding claim: the proposition that some primitive state is actual at some instant of time does not determine which complete individualistic profile is true at that time. The state-space-first picture requires additional fundamental entities whose pattern is detailed by the relevant propositions, such as properties of and relations among states. So, whereas individualistic states determine complete individualistic profiles in isolation, primitive states determine these profiles only collectively. While individualistic states may have some resemblance to possible worlds, primitive states clearly do not. (I say more about the modal status of primitive states near the end of this paper.)

\footnotetext{
${ }^{27}$ (Bacon, 2019) develops a version of state-space-first metaphysics, albeit for somewhat different philosophical purposes: he deploys state-space theoretic reasoning to illustrate a general strategy aimed at the more ambitious goal of articulating a purely qualitative account of the fundamental - one that dispenses with individuals in a broader logical sense of first-order individuals. By contrast, the individualism to which state-space-first metaphysics is opposed is the view that fundamental physical reality consists in individuals of a more specific, physical sort: objects like elementary particles. My kind of state-space-first metaphysics is consistent with the more general kind of individualism that is the target of Bacon's work. The view proposed in (North, 2009) merely entails that state space is a real physical space, but not that it is fundamental.
} 
To identify the physical entities whose pattern is detailed in the relevant fundamental propositions, we need to specify the state-space-first view in more detail. An immediate question concerns the ontological category of primitive states. This is a subtle choice point for the state-space-first approach. One option is state-space substantivalism, the view that primitive states are first-order individuals. In addition to primitive states, this view posits instants of time: first-order individuals that bear a fundamental dyadic actuality relation to states. This relation can be paraphrased as "... is actual at..." and satisfies the constraint that, necessarily, for every instant of time $t$ there is some state $s$ such that $s$ is actual at $t .{ }^{28}$ An alternative view conceives of primitive states as dyadic relations between instants of time and a single first-order individual - 'the world' - such that necessarily, for every instant of time, there is some relation that the world bears to that instant. ${ }^{29}$ Another alternative regards primitive states as monadic properties of instants of time such that necessarily, for every instant of time there is some such property instantiated by it. ${ }^{30}$ Although I focus on the substantivalist option in this paper, my main conclusions do not depend on this choice. The core of the view I propose is that primitive states are fundamental physical entities that figure in the fundamental propositions that entail complete individualistic profiles. This view is indifferent to whether states are conceived of as first-order individuals, first-order relations between the world and instants of time, or as monadic properties of instants of time. ${ }^{31}$

In addition to states, times, and the actuality relation, we also need to posit properties and relations that confer symplectic structure on primitive states and temporal structure on instants of time. I will not say more about these ontological primitives here, except for the following. First, the literature contains successful proposals for an intrinsic account of temporal structure. ${ }^{32}$ Second, an intrinsic account of the properties and relations that confer symplectic structure is tantamount to the nominalization of symplectic geometry. This project is beyond the scope of this paper; for present purposes, it will suffice to refer to the symplectic structure in mathematical terms.

A few paragraphs ago, I noted that the complete individualistic profile at an instant of time is determined by a proposition that does not just say which primitive state is actual at that time, but that also details the pattern in certain properties of and relations among primitive states. Are the properties and relations that confer symplectic structure sufficient for this task?

The answer is no. State spaces of classical particle worlds can agree about the entities

\footnotetext{
${ }^{28}$ The explicit quantification over instants of time means that state-space substantivalism has a distinctively B-theoretic flavour. It is fairly straightforward to articulate an A-theoretic version of the view - a version which does not quantify over instants of time - by replacing the actuality relation with a monadic property '...is actual', and introducing primitive propositional tense operators such as 'it will always be the case that...', 'it will at some time be the case that...' and so on.

${ }^{29}$ The A-theoretic version of this view regards primitive states as monadic properties of the world such that necessarily, the is always some such property instantiated by the world.

${ }^{30}$ The A-theoretic version of this view conceives of states as zero-place properties: i.e. propositions such that necessarily, there is always some such proposition that is true.

${ }^{31}$ See (Bacon, 2019) for a discussion of some foundational issues raised by this choice point.

${ }^{32}$ For example, Arntzenius and Dorr (2012, pp. 223) proceed in terms of temporal betweenness and temporal congruence; cf. Field (1980, Chpt. 6).
} 
that confer symplectic structure while disagreeing about particle number. A twelvedimensional phase space can be used to characterize the state space of two particles in three spatial dimensions as much as it can be used to describe the state space of three particles in two spatial dimensions. Complete individualistic profiles aren't determined by symplectic structure alone; additional physical structure is needed. As I will explain shortly, central among these primitives are relations among states that correspond to state-space symmetries.

In this section, I contrasted individualism - the default ontology for classical particle mechanics - with the state-space-first approach. The next task is to introduce the ontological primitives whose patterns determine complete individualistic profiles. There will be no more than four primitives, two of which correspond to state-space symmetries. This will demonstrate that state-space-first metaphysics is an instance of symmetry fundamentalism.

\section{Individualistic Profiles from Primitive States}

I will introduce the relevant primitives by their individualistic paraphrases; but it is important to emphasize that these individualistic characterisations are not metaphysical definitions: as primitives, these items cannot be characterized or defined in more basic terms. Any individualistic characterisation is a mere conceptual crutch that we must ultimately kick away to be replaced by a full account of individualistic talk in statespace-first terms. This is a familiar aspect of metaphysical theorizing: when making unfamiliar metaphysical posits, we need to defend them by presenting a theory in which they figure and by showing how that theory is inferentially and explanatorily connected to notions we already understand. This is precisely what I will do momentarily: I will explain how the ontological primitives I introduce figure in the fundamental propositions that entail complete individualistic profiles.

I already mentioned some of the fundamental posits of state-space-first metaphysics. On the state-space substantivalist version of this approach, we posit states, instants of time, the actuality relation, as well as symplectic and temporal structure. (For ease of exposition, in this section I will refer to primitive states simply as 'states'.) There are four additional primitives; here are the first three: ${ }^{33}$

1. Position-Almost-Sameness. A dyadic relation. Relates two states iff they agree about the momenta of all particles and about the positions of all but one particle.

2. Momentum-Almost-Sameness. A dyadic relation. Relates two states iff they agree about the positions of all particles and about the momenta of all but one particle.

3. Degeneracy. A monadic property. Picks out a 6-dimensional subspace of state space consisting of states at which all particles have the same position and the same momentum.

\footnotetext{
${ }^{33}$ The following is inspired by (Dorr, 2018).
} 
Before we can introduce the fourth primitive, we need to do some work using the other primitives. There are four steps.

First, position-almost-sameness picks out a range of subspaces such that any two elements are position-almost-same; similarly for momentum-almost-sameness. Call these subspaces the position-almost-same subspaces and the momentum-almost-same subspaces, respectively.

Second, observe that the points in a given position- or momentum- almost-same subspace differ about the position or momentum of the same particle, respectively. The reason is that two states $y, z$ can be (say) position-almost-same to some state $x$ without being position-almost same to each other. The fact that $x, y$ and $x, z$ are position-almostsame, respectively, implies the position-almost-sameness of $y$ and $z$ only if the differences between $x, y$ and $x, z$ concern the same particle.

The position- and momentum-almost-same subspaces are characterized in terms of certain specific state-space symmetries: the position and momentum shift flows, i.e. the transformations which (on the individualistic picture) correspond to shifts of individual particles in position or momentum. Recall: a phase-space flow is a family of curves such that every phase space point is intersected exactly once by exactly one curve in this family. For any triple of spatial coordinate axes, there is a triple of phase space flows that corresponds to shifting some specific particle along these three axes. Of course, there is a continuum infinity of such triples - one for every coordinate system - each of which is equally well-suited for describing the almost-same subspaces. This is as it should be: differences between coordinate systems are a paradigm instance of mathematical differences without corresponding metaphysical distinctions.

Here is the third step. We can define physical counterparts of level surfaces of coordinate functions in terms of almost-same subspaces. The level surfaces of a coordinate function on phase space are regions of phase space in which that function is of constant value. Level surfaces may be familiar from hiking maps where they are known as contour lines: regions of constant height. See figure 5 for an illustration.

The physical counterparts of level surfaces are what I refer to as position and momentum foliations. We can give them the following individualistic characterization: any two states on a leaf of a position foliation agree about the position of some particle while possibly disagreeing about the positions of all other particles and about the momenta of all particles; similarly, any two states on a leaf of a momentum foliation agree about the momentum of some particle while possibly disagreeing about the momenta of all other particles and about the positions of all particles. Position and momentum foliations are equivalence relations with leaves as equivalence classes; and two states $x, y$ are on the same leaf of (say) a position foliation $Q$ iff $Q x y$.

Position and momentum foliations are mathematically represented by the level surfaces of phase-space coordinate functions. The subspace of phase space that corresponds to a given leaf of a position foliation is the subspace such that, for any two phase space points $s, s^{\prime}$ in this subspace, for some particle, for every triple of spatial coordinate functions of that particle, and for some triple of level surfaces of these functions, $s$ lies on those level surfaces iff $s^{\prime}$ does. Differently put: any two points in a subspace of phase space that corresponds to a given leaf of a position foliation agree about the level surfaces of all 


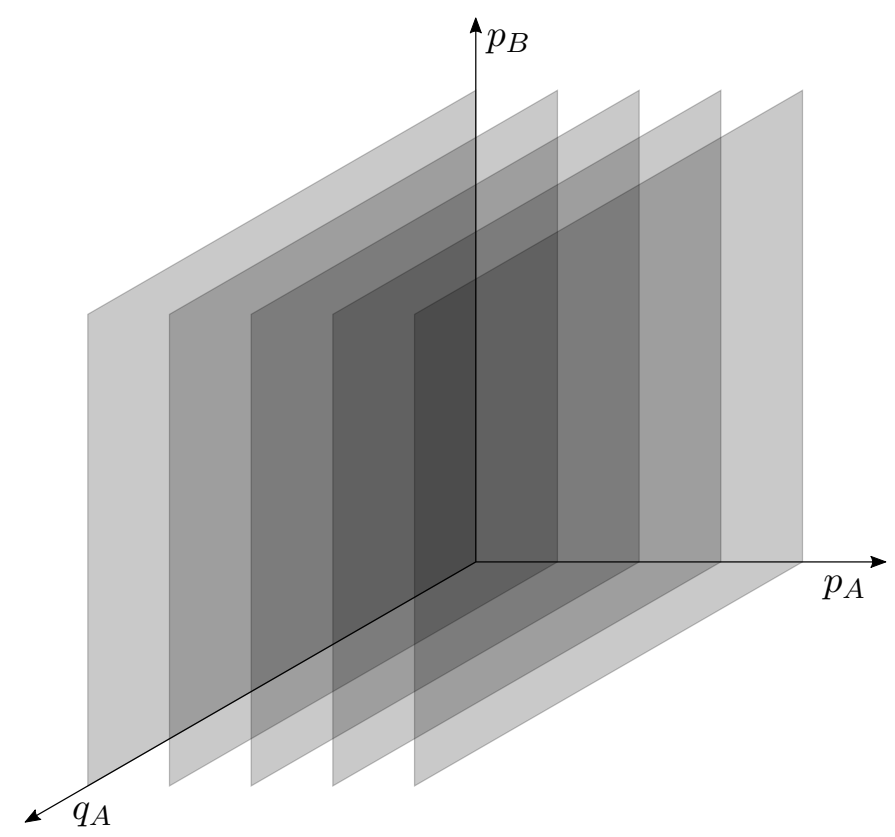

Figure 5: An illustration of the phase space of two particles (here labeled by $A$ and $B$ ) in one spatial dimension. Axes are labeled by the coordinate functions for the position of particle $A\left(q_{A}\right)$, the momentum of $A\left(p_{A}\right)$, and the momentum of $B\left(p_{B}\right)$; the axis corresponding to the position of $B\left(q_{B}\right)$ is suppressed. The shaded regions are segments of the level surfaces of $p_{A}$. (More precisely: the shaded regions are the intersections of the three-dimensional level surfaces of $p_{A}$ with some specific three-dimensional level surface of $\left.q_{B}\right)$.

spatial coordinate functions of some particle.

The goal is now to replace the individualistic characterization of position and momentum foliations with a definition in terms of position- and momentum-almost-sameness. This definition involves the symplectic structure of state space. As I said before, for present purposes it will be sufficient to refer to this structure in mathematical terms. The key thing to note, then, is that position and momentum foliations can be defined in terms of the position- and momentum-almost-sameness relations at the level of mathematical description: that is, the level surfaces of a given coordinate function can be defined in terms of a specific phase space flow.

The mathematical details of this definition are quite involved and need not concern us here. ${ }^{34}$ It will suffice to note that the definition is based on four mathematical

\footnotetext{
${ }^{34}$ The details go as follows. Let $(M, \omega)$ be a symplectic manifold, $p$ a smooth real-valued function on $M, \exp _{x}: T_{x} M \rightarrow M, v \mapsto \exp _{x}(v)$ the exponential map and $d$ the exterior derivative. The level surfaces of $p$ are encoded in the exterior derivative $d p$ of $p$; and $d p$ uniquely corresponds to the phase space flow

$$
\begin{aligned}
\gamma: M \times \mathbb{R} & \rightarrow M, \\
(x, t) & \mapsto \gamma(x, t)=\exp _{x}\left(\left.t \omega^{-1}(d p)\right|_{x}\right)
\end{aligned}
$$


observations: first, every vector field has a flow; for example, figure 6 shows the gradient vector field of a momentum coordinate function. Its flow takes us along paths of greatest increase of this function. Second, there is a one-to-one correspondence between the level

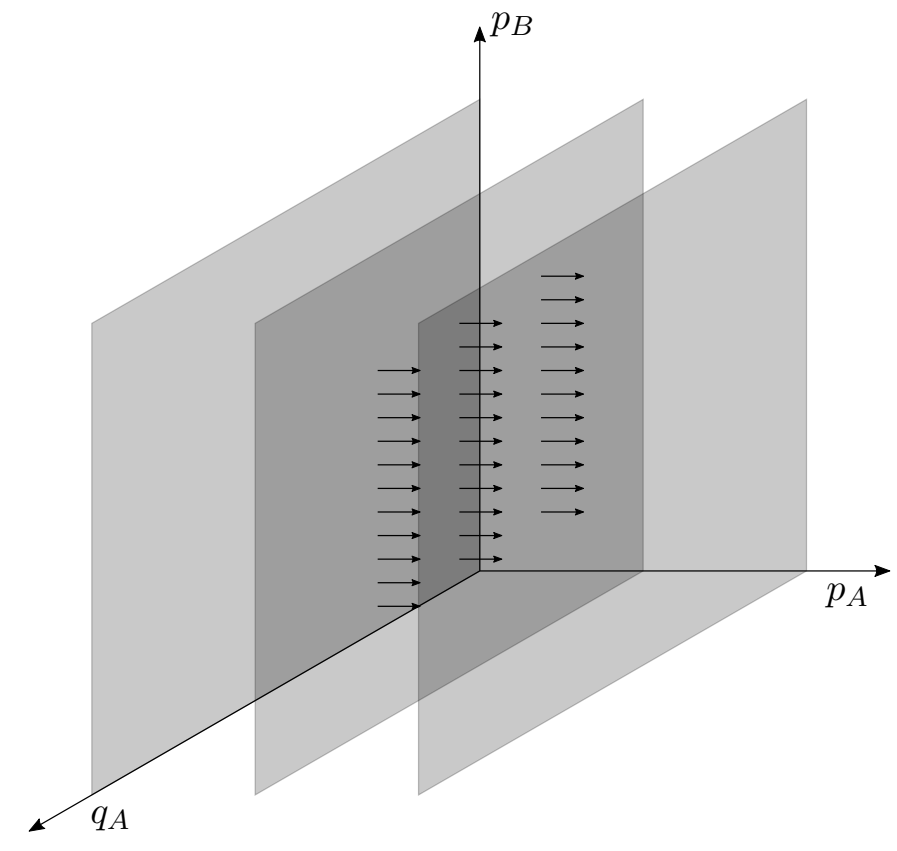

Figure 6: The gradient of $p_{A}$ is everywhere at a right angle to the level surfaces of $p_{A}$ and its length at a point is proportional to the rate of change of $p_{A}$ around that point.

surfaces of this coordinate function and its gradient: the level surfaces uniquely determine the gradient and vice versa. Third, for technical reasons, phase spaces generally do not admit of a gradient vector field, but instead of what's referred to as a Hamiltonian vector field: a vector field that differs from the gradient in that it does not point in the direction of greatest increase of a function but rather along the level surfaces of that function, as illustrated in figure $7 .^{35}$ But Hamiltonian vector fields are similar to gradients in the

where $\omega^{-1}(d p)$ is the Hamiltonian vector field of $p$. Since $\omega^{-1}(d p, d p)=0$, i.e. the rate of change of $p$ under $\gamma$ vanishes, $\gamma$ is confined to the level surfaces of $p$. Moreover, there is a smooth function $q$ (unique up to an arbitrary additive constant) such that $\omega^{-1}(d q, d p)=1$. If $p$ is the momentum coordinate function of some particle along some axis, then $q$ is the corresponding position coordinate. For the purposes of ontological theorizing, we can read the identity in (1) as stating a definition of $d p$ (corresponding to an aspect of a momentum foliation) in terms of $\gamma$ (corresponding to an aspect of a position-almost-same subspace).

To be sure, the identity in (1) by itself does not require this reading: there is nothing in the mathematics that suggests an asymmetry between the two sides of the equality sign. However, we are presently concerned with ontological theorizing about physics. As I understand this project here, it consists in the articulation of hypotheses about physical reality in a way that may involve drawing metaphysical distinctions between mathematically equivalent entities; cf. (Maudlin, 2018).

${ }^{35}$ The reason is that phase spaces generally do not admit a Riemannian metric, a mathematical entity that links the level surfaces of a function to gradient vector fields. Instead, as already mentioned, phase spaces admit of a symplectic form - an entity which links the level surfaces of any smooth real-valued 


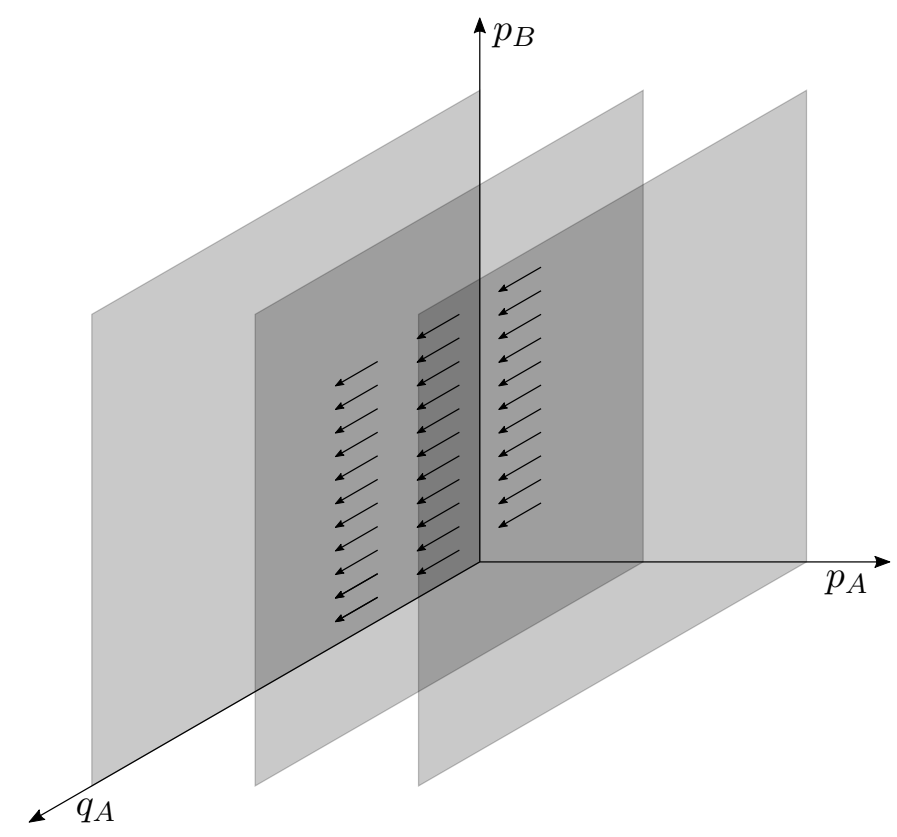

Figure 7: The Hamiltonian vector field of $p_{A}$ everywhere points along the level surfaces of $p_{A}$. Its length at a point is proportional to the rate of change of $p_{A}$ around that point.

following respect: for every Hamiltonian vector field there is some unique collection of level surfaces and a unique flow to which it corresponds. Fourth, the level surfaces of the position coordinate of some particle in some direction uniquely correspond to the Hamiltonian vector field whose flows are shifts in the momentum coordinate of that particle in that direction; similarly, the level surfaces of the momentum coordinate of some particle in some direction uniquely correspond to the Hamiltonian vector field whose flows are shifts in the position coordinate of that particle in that direction. This is illustrated in figure 8 .

The result is the following: at the level of mathematical description, position foliations are definable in terms of momentum-almost-sameness, and momentum foliations are definable in terms of position-almost-sameness - in both cases, together with the symplectic structure of state space. ${ }^{36}$

The fourth step is to make use of the degeneracy property. The idea is that degenerate states serve to define surrogates for position and momentum values. We begin with defining the relations of hereditary position-/momentum almost-sameness as the transitive closures of the position-/momentum-almost-sameness relations. In other words: any two hereditarily position-almost-same states agree about the momenta of all particles but

function with some specific Hamiltonian vector field.

${ }^{36}$ The definitions of position-foliations in terms of momentum-almost-sameness and of momentumfoliations in terms of position-almost-sameness are a reflection of the correspondence between position and momentum degrees of freedom, also known as canonical conjugation, that is one of the characteristic features of the Hamiltonian formulation of classical mechanics. 


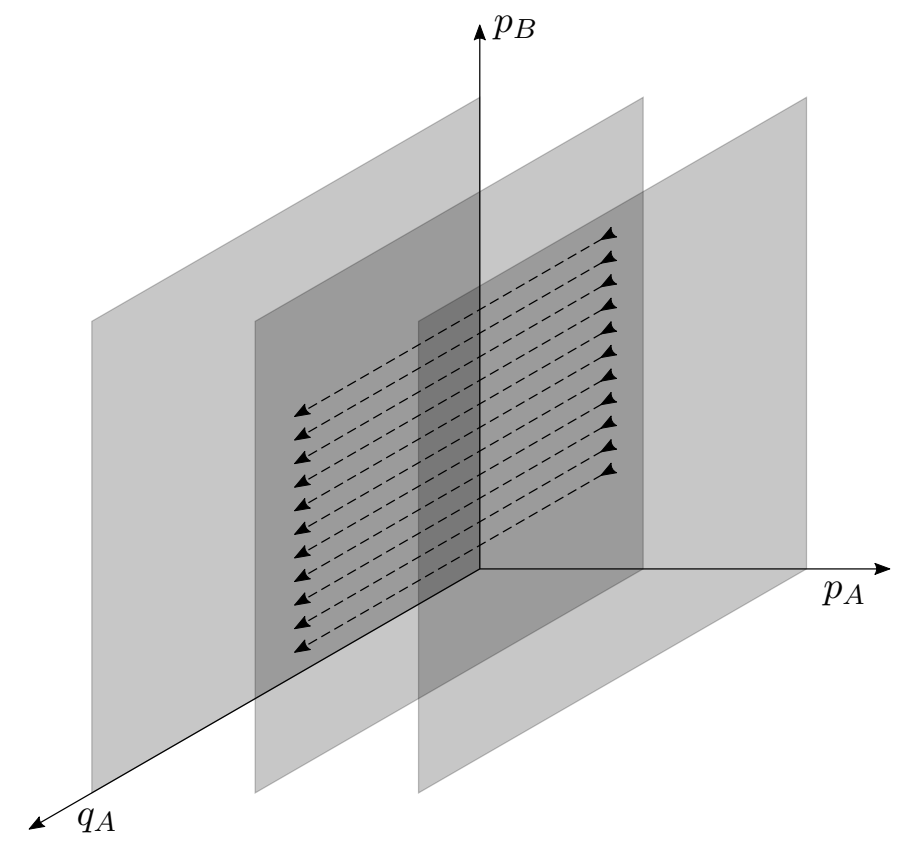

Figure 8: The flow lines (dotted) of the Hamiltonian vector field of $p_{A}$.

possibly disagree about particle positions, and any two hereditarily momentum-almostsame states agree about the positions of all particles but possibly disagree about particle momenta. Next, let a position-degenerate subspace be a subspace of points hereditarily momentum-almost-same to some degenerate point and let a momentum-degenerate subspace be a subspace of points hereditarily position-almost-same to some degenerate point. Individualistically speaking, states in position-degenerate subspaces agree about the spatial location at which all particles coincide but can disagree about particle momenta, and states in momentum-degenerate subspaces agree about the momentum value instantiated by all particles but can disagree about particle positions. ${ }^{37}$

The strategy is now to identify individualistic quantification over particles with quantification over position- and momentum-foliations, and quantification over position/momentum values with quantification over position-/momentum-degenerate subspaces. Here is a first pass at this idea: at $t$, some particle has spatial location $q$ iff there is a primitive state $x$ such that $x$ is actual at $t, q$ is a position-degenerate subspace and there is a position foliation $Q$ such that $Q x z$ for every point $z$ in $q$.

This is not quite right. Consider the claim that, at $t$, some particle has spatial location $q$ and momentum $p$. The first pass suggests that this claim is equivalent to the proposition that

1. there is a primitive state $x$ such that $x$ is actual at $t$ and

\footnotetext{
${ }^{37}$ When the symplectic manifold that corresponds to state space is a cotangent bundle, positiondegenerate subspaces correspond to certain specific fibres in this bundle and momentum-degenerate subspaces to certain specific sections.
} 
2. $q$ is a position-degenerate subspace and $p$ is a momentum-degenerate subspace and

3. for some position foliation $Q$ and some momentum foliation $P$ : $Q x y$ for every $y$ in $q$ and $P x z$ for every $z$ in $p$.

But this is incorrect. The individualistic proposition attributes position and momentum values to the same particle. However, there is no corresponding structure in its proposed fundamental counterpart: something which serves as the ground for individualistic attributions of positions and momenta, an entity in virtue of which the fundamental structure of the classical symmetry-fundamentalist world is rich enough to give rise to non-fundamental individualistic profiles. What is missing, in individualistic terms, is a fundamental notion which ensures that position- and momentum-foliations - the fundamental surrogates for position and momentum values - belong to the same particle. The state-space-first metaphysician needs to make an additional posit, and one straightforward option is the following relation:

4. Particle-Sameness. A dyadic relation which relates a position- and momentum-foliation iff they belong to the same particle.

The role played by this relation is analogous to imposing a convention that details which phase-space coordinates belong to which particle. For an $N$-particle system, the standard convention is that the first $N$ triples of phase-space coordinates of a given phase-space point are the position coordinates and the last $N$ triples are the momentum coordinates, so that coordinates $1,2,3$ and $N+1, N+2, N+3$ are the position and momentum coordinates, respectively, of the same particle. The relation of particle-sameness is a fundamental posit that captures just this kind of structure at the fundamental level.

Let me stress again that neither the label nor the individualistic characterization of particle-sameness should be read as a metaphysical definition of this relation, or as implying that a state-space-first metaphysics must ultimately reintroduce particles at the fundamental level. Just like almost-sameness and degeneracy, particle-sameness is fundamental and so cannot be defined in more fundamental terms. Nonetheless, this fact does not prevent us from using individualistic language to pin down these fundamental notions - as long as we ultimately succeed in vindicating such individualistic characterizations.

Indeed, this is precisely what particle-sameness allows us to achieve. The modified proposal is as follows: at $t$, some particle is at position $q$ with momentum $p$ iff

1. there is a primitive state $x$ such that $x$ is actual at $t$ and

2. $q$ is a position-degenerate subspace and $p$ is a momentum-degenerate subspace and

3. for some position foliation $Q$ and some momentum foliation $P$ such that $P$ and $Q$ belong to the same particle: $Q x y$ for every $z$ in $q$ and $P x z$ for every $z$ in $p$.

Let me illustrate this proposal for a world containing two particles in one spatial dimension. (For ease of exposition, I will label these particles as $A$ and $B$. Nothing 
of metaphysical significance hangs on these labels.) The state space of this world is four-dimensional. Figure 9 shows a projection of this space onto a three-dimensional subspace in which the position of particle $B$ is fixed. In other words, this diagram depicts some specific leaf of a position-foliation. There are now three things to explain.

First of all, the degenerate states form a two-dimensional subspace. Since one dimension is suppressed in figure 9, the degenerate states form a one-dimensional subspace. Since no degenerate state in this diagram can differ about the fixed position of particle $B$, this line must intersect the $q_{A^{-}}$axis at a right angle. Moreover, states in this subspace agree in the momenta of both particles, so this line is at a $45^{\circ}$ angle to both the $p_{A}$ and the $p_{B}$ axes. If $a$ is the state at which $q_{A}$ matches $q_{B}$, then this subspace is given by the dashed line.

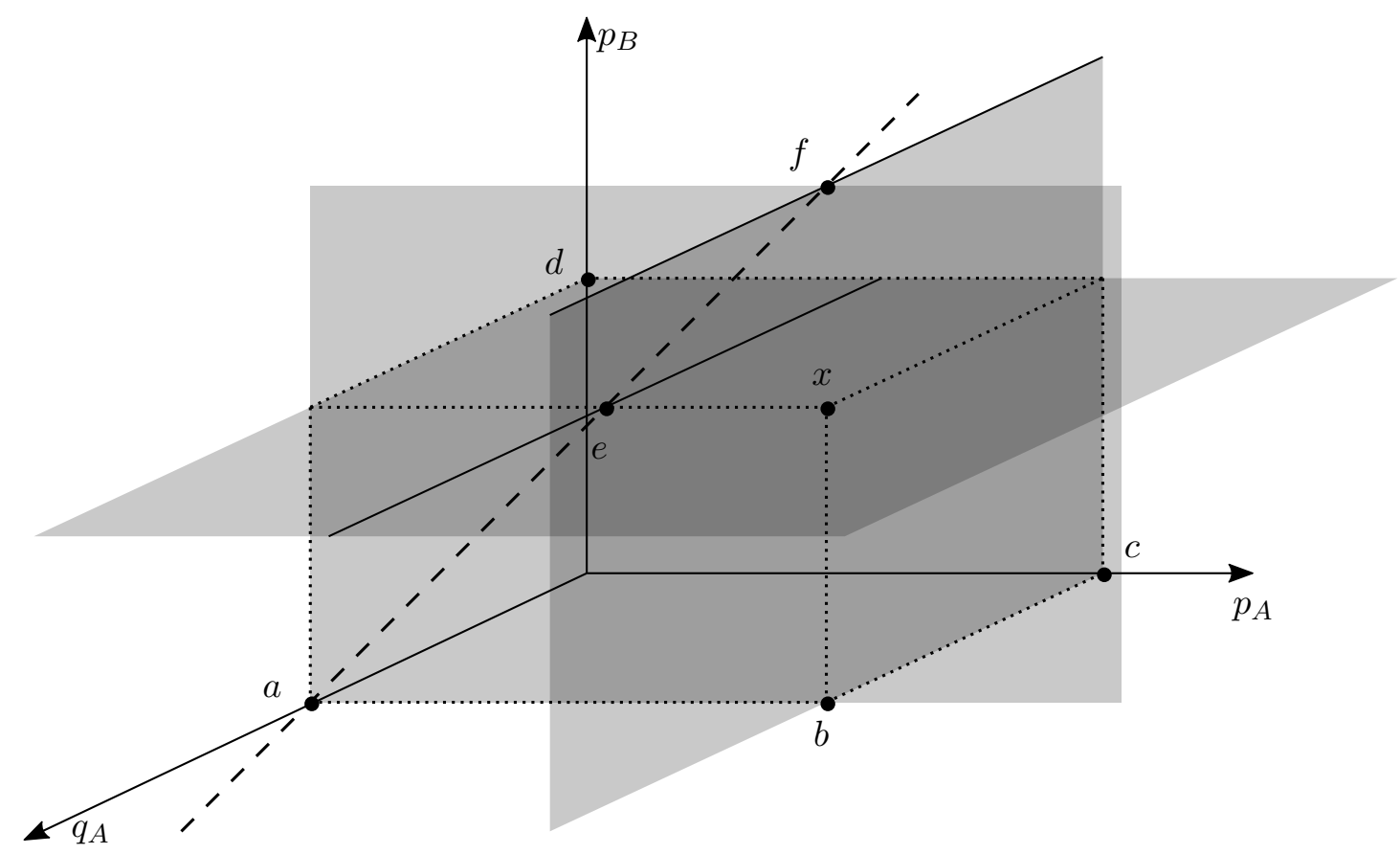

Figure 9: Position- and momentum-degenerate subspaces as well as position- and momentum-foliations of a two-particle world in one spatial dimension.

Second, position- and momentum-degenerate subspaces are two-dimensional subspaces of state space, while the leaves of position- and momentum-foliations (or position- and momentum-leaves, for short) are three-dimensional subspaces. Position- and momentumleaves are projected down to two-dimensional surfaces shown in this diagram: the surfaces spanned by dex and $b c x$ are leaves of momentum-foliations, respectively, and $a b x$ is a leaf of a position foliation. Since the only differences in position between states in this diagram are differences in the position of $A$, momentum-degenerate subspaces are one-dimensional, such as the solid lines intersecting states $f$ and $e$. By contrast, position-degenerate subspaces in this diagram are two-dimensional surfaces that (due to the suppression of one dimension) coincide with leaves of position foliations. An example 
is the surface spanned by $a b x$.

Finally, we can verify the above account of individualistic propositions. At state $x$, the position of particle $A$ is the position-degenerate subspace that coincides with $a b x$ and its momentum is the momentum-degenerate subspace through $f$ : the former coincides with the position-leaf spanned by $a b x$ and has the same $q_{A}$-coordinate as $x$, the latter is completely within the momentum-leaf spanned by $b c x$ and has the same $p_{A}$-coordinate as $x$. Similarly, the momentum of particle $B$ is the momentum-degenerate subspace intersecting $e$, which lies completely within the momentum-leaf spanned by dex and has the same $p_{B}$-coordinate as $x$. The relation of particle-sameness is not pictured in this diagram; think of it as fixing the particle labels of the coordinate axes.

The proposal just illustrated is sufficient for specifying the fundamental propositions that entail the individualistic propositions. Complete individualistic profiles are conjunctions of existentially quantified claims of the form 'there is some particle at position $q$ with momentum $p$ '. As we just saw, each of those conjuncts is equivalent to a fundamental proposition about primitive states and the properties of and relations among states. We have therefore met the key challenge facing the state-space-first metaphysician: to specify fundamental propositions (about primitive states and their relations) such that every complete individualistic profile is entailed by some such proposition. On the resulting view, the complete individualistic profile of the world at some instant of time is equivalent to a proposition that details which primitive state is actualized at that time as well as the pattern in the primitive physical structure on state space. Here is a list of the ontological primitives of state-space substantivalism:

\begin{tabular}{|c|c|}
\hline \multicolumn{2}{|c|}{ State-Space Substantivalism } \\
\hline \hline Ontological Primitive & Type \\
\hline Primitive states. & A collection of first-order individuals. \\
\hline Instants of time. & A collection of first-order individuals. \\
\hline Actuality. & $\begin{array}{c}\text { A single dyadic relation that takes one } \\
\text { primitive state and one instant of time as } \\
\text { arguments. }\end{array}$ \\
\hline $\begin{array}{c}\text { The properties and relations that confer } \\
\text { symplectic structure on primitive states. }\end{array}$ & $\begin{array}{c}\text { A collection of properties of and relations } \\
\text { among primitive states. }\end{array}$ \\
\hline $\begin{array}{c}\text { The properties and relations that confer } \\
\text { temporal structure on instants of time. }\end{array}$ & $\begin{array}{c}\text { A collection of properties of and relations } \\
\text { among instants of time. }\end{array}$ \\
\hline $\begin{array}{c}\text { Position- and } \\
\text { momentum-almost-sameness. }\end{array}$ & $\begin{array}{c}\text { Two dyadic relations among states. } \\
\text { Degeneracy. }\end{array}$ \\
\hline Particle-sameness. & $\begin{array}{c}\text { A single monadic property of states. } \\
\text { that takes dyadic recond-order relations among states } \\
\text { as arguments. }\end{array}$ \\
\hline
\end{tabular}

\footnotetext{
${ }^{38}$ In the language of relational type theory, a first-order property is an entity of type $\langle e\rangle$; a first-order relation is an entity of type $\langle e, e\rangle$; and a second-order relation among dyadic first-order relations is an entity of type $\langle\langle e, e\rangle,\langle e, e\rangle\rangle$; where $e$ is the type of individuals, \langle\rangle the type of propositions and for any types $\tau_{1}, \ldots, \tau_{n}, n \geq 0,\left\langle\tau_{1}, \ldots, \tau_{n}\right\rangle$ is a type; nothing else is a type. See (Dorr, 2016, pp. 49-52) for details.
} 
State-space-first proposals like state-space substantivalism give symmetry fundamentalism a precise metaphysical underpinning. As mentioned earlier, state-space symmetries - phase space flows that preserve the symplectic form - correspond to physical entities, the almost-sameness relations. State-space-first proposals of this kind therefore make good on the idea that mathematical symmetries correspond to fundamental aspects of physical reality, whereas things like particles are ontologically derivative of these aspects.

But you might think that we could have achieved this much more straightforwardly: simply by pointing to the mathematical symmetry transformations and declaring that it is the physical entities to which these transformations correspond-whatever they might be - that figure in the symmetry fundamentalist ontology. The underlying thought is that, when specifying an ontology for a physical theory, it is sufficient to identify certain aspects of the mathematical formulation of that theory as the representationally significant ones - those that are taken to in some sense 'correspond to' parts of physical reality. On this approach, there is no requirement that the relevant physical entities be characterized independently of their mathematical representations.

The approach I've taken in this paper is more ambitious. I have characterized the physical entities that correspond to mathematical symmetry transformations in purely physical terms: that is, in terms which make transparent that they are distinct from their mathematical representations and which explains the suitability of those mathematical representations as tools for characterizing the fundamental ontological inventory of state-space-first metaphysics. ${ }^{39}$

The advantages of this more ambitious approach should now be clear. First of all, we saw that the state-space structure that goes beyond symplectic structure can be captured in terms of just four ontological primitives: two almost-sameness relations, the monadic degeneracy property, as well as the relation of particle-sameness. This reveals that the physical structure of state space in classical particle mechanics is significantly simpler than its mathematical characterization might suggest - a fact that we would likely have missed had we not gone beyond the mathematical formalism in our ontological theorizing.

Second of all, the metaphysical insight afforded by our account is unlikely to be matched by the more modest approach. The reason is that symmetry fundamentalism is a particularly puzzling claim. We are familiar with fundamentalism about Euclidean space as a certain specific view about the structure of fundamental physical space. By contrast, fundamentalism about state-space symmetries is an altogether less familiar and more puzzling thing. Declaring that a range of symmetry transformations are representationally significant and that they 'correspond to' purely physical entities does not allow us to see even dimly what these purely physical entities could be. We might

\footnotetext{
${ }^{39} \mathrm{My}$ approach is inspired by nominalistic constraints on ontological theorizing, according to which physical ontology must be specified without invoking mathematical entities at all; cf. (Field, 1980; Arntzenius and Dorr, 2012; Chen, 2018). However, limitations of space prevent me from fully complying with nominalistic strictures in this paper. Instead, I have provided a nominalistic characterization of merely one part of the ontology: the part concerned with the fundamental items corresponding to mathematical symmetry transformations.
} 
even run the risk of being profoundly misunderstood: to some, it may sound like we're proposing a kind of pythagoreanism, according to which physical reality consists (at least in part) of mathematical objects. Specifying the relevant ontological primitives in purely physical terms - as I did in this paper - is a way to avoid problems of this kind.

Finally, let me briefly dispel a worry that some readers might have about the kind of ontology presented in this paper: since states seem to have modal characteristics, the view that state space is fundamental looks appears to imply a kind of modal realism, according to which non-actualized, merely possible states are every bit as real as the actualized state.

Contrary to appearances, state-space substantivalism does not have this implication. As already noted, individualistic states certainly do have a modal flavour: for every proposition about the positions and momenta of particles statable in the individualistic theory, complete individualistic profiles entail either it or its negation. Intuitively, an individualistic state can be thought of as representing an instantaneous way the world could be - much like a possible world. But these modal characteristics of individualistic states are not inherited by their counterparts in the state-space-first ontology, the primitive states. As I argued earlier, the fact that the world is in some primitive state at some instant of time by itself does not fix the complete individualistic profile of the world at that instant; for that, we also need to know the relationships between that primitive state and other primitive states. According to state-space substantivalism, the space of primitive states is simply the fundamental space in which the history of the world unfolds.

To be sure: the fundamental state space does obey the constraint, familiar from the individualistic setting, that for any instant of time, the world can be in no more and no less than one state at that instant. But whereas in the individualistic theory, this constraint follows from the fact that individualistic states are maximally specific and exhaustive, the state-space fundamentalist theory must add this constraint as a basic law: there is nothing about the nature of primitive states that guarantees mutual exclusivity. Any kind of modal status that this constraint may be thought to confer on primitive states has its source in this basic law rather than in some putative intrinsic modal property of primitive states.

\section{Conclusion}

In this paper, I developed an ontology for classical particle mechanics that gives symmetry fundamentalism a precise metaphysical underpinning - the view that symmetries correspond to fundamental aspects of physical reality, whereas the entities we might ordinarily have thought of as the fundamental building blocks of the world, such as particles or fields, are ontologically derivative of those aspects. Our investigation illustrates the way in which symmetry-fundamentalist ontological theorizing does justice to the paradigm shift towards symmetry techniques in modern physics and serves as a guide for articulating symmetry-fundamentalist ontological proposals for quantum theory, a 
project I carry out elsewhere. ${ }^{40}$

Given the absence of coherent ontological alternatives, individualism has so far been the default option for the ontology of classical particle mechanics. One result of this paper is that this is no longer the case: the foregrounding of symmetries in metaphysical theorizing led us to state-space-first views as a range of coherent ontological alternatives to individualism. It may therefore be worth reopening the question whether individualism should be regarded as the superior metaphysics for classical particle mechanics. At the very least, a thorough and systematic evaluation of the strengths and weaknesses of state-space-first proposals vis-à-vis individualism might improve our grasp of why the latter is preferable as an ontology for classical particle mechanics. This is a task for another day.

Finally, there are two general lessons to be drawn from this paper. First, I hope to have shown what we can achieve when we let go of our preconceptions about what the world must be like fundamentally. There is lots of progress to be made by taking inspiration from physicists and from the mathematical formulations of physical theories.

Second, our investigation demonstrates that taking physics itself as the starting point for metaphysical theorizing isn't a way of making metaphysics or its methods redundant. Quite the contrary: doing so showcases the power of metaphysics as a tool for exploring the full range of ontological options for fundamental physics.

\section{Acknowledgements}

I would like to thank David Albert, Claus Beisbart, Daniel Berntson, Alex Blum, Ellie Cohen, Neil Dewar, Andreas Ditter, Steven French, Wolfgang Hasse, Meir Hemmo, Josh Hunt, Caspar Jacobs, James Ladyman, Vassilis Livanios, Alex Meehan, Jill North, Daniel Olson, Pablo Ruiz de Olano, Sondre Solstad, Trevor Teitel, Barbara Vetter, three anonymous referees, as well as audiences at the XXI International Summer School in Philosophy of Physics in Urbino, at the Munich Center for Mathematical Philosophy, at the Max-Planck-Institute for the History of Science in Berlin, at the 2018 Society for the Metaphysics of Science Conference in Milan, at the University of Bristol, at the Free University Berlin, at the 2019 Eastern APA in New York City, as well as at the University of Oxford for helpful comments and discussions. Above all, I owe thanks to Cian Dorr, Hans Halvorson, Boris Kment, Tim Maudlin, and Gideon Rosen.

This work was made possible in part through the generous financial support of the Thomas und Ulla Kolbeck Stiftung, Germany.

I acknowledge that Princeton University is on the traditional territory of the Lenape Nations and I recognize that this territory continues to be home to many Algonkian peoples. I give blessings and thanks to the Lenape people and Lenape Nations in recognition that this work has been carried out on their indigenous homelands.

\footnotetext{
${ }^{40}$ (Schroeren, 2020).
} 


\section{References}

Arenhart, J. R. B. and Bueno, O. (2015). Structural realism and the nature of structure. European Journal for Philosophy of Science, 5(1):111-39.

Arntzenius, F. and Dorr, C. (2012). Calculus as geometry. In Arntzenius, F., editor, Space, Time, and Stuff, chapter 8, pages 213-78. Oxford: Oxford University Press.

Bacon, A. (2019). Is reality fundamentally qualitative? Philosophical Studies, 176(1):25995.

Ballentine, L. E. (2000). Quantum Mechanics: A Modern Development. London: World Scientific.

Brading, K. and Castellani, E. (2003). Symmetries in Physics - Philosophical Reflections. Cambridge: Cambridge University Press.

Brading, K., Castellani, E., and Teh, N. (2017). Symmetry and Symmetry Breaking. In Zalta, E. N., editor, The Stanford Encyclopedia of Philosophy. Metaphysics Research Lab, Stanford University, winter 2017 edition.

Castellani, E. (1998). Interpreting Bodies. Princeton: Princeton University Press.

Chen, E. K. (2018). The Intrinsic Structure of Quantum Mechanics. PhilSci Archive. Article ID: 15140 .

Dasgupta, S. (2009). Individuals: an essay in revisionary metaphysics. Philosophical Studies, 145(1):35-67.

Dasgupta, S. (2016). Symmetry as an Epistemic Notion (Twice Over). British Journal for the Philosophy of Science, 67(3):837-878.

Dorr, C. (2010). Review of Every Thing Must Go: Metaphysics Naturalized. Notre Dame Philosophical Reviews. https://ndpr.nd.edu/news/ every-thing-must-go-metaphysics-naturalized/, accessed 22 April 2020.

Dorr, C. (2016). To Be F Is To Be G. Philosophical Perspectives, 30(1):39-134.

Dorr, C. (2018). A Way of Getting Rid of Things: Higher-order Languages, Priorian Nominalism, and Nihilism. http://www.nyu.edu/projects/dorr/handouts/Nihilism. pdf, accessed 22 April 2020.

Earman, J. (2003). Rough guide to spontaneous symmetry breaking. In Brading, K. and Castellani, E., editors, Symmetries in Physics-Philosophical Reflections, pages 335-46. Cambridge: Cambridge University Press.

Field, H. (1980). Science Without Numbers. Princeton: Princeton University Press.

Folland, G. (2015). A Course in Abstract Harmonic Analysis. Boca Raton: CRC Press, 2nd edition. 
French, S. (2010). The interdependence of structure, objects and dependence. Synthese, 175(1):89-109.

French, S. (2014). The Structure of the World: Metaphysics and Representation. Oxford: Oxford University Press.

French, S. and Ladyman, J. (2003). Remodelling Structural Realism: Quantum Physics and the Metaphysics of Structure. Synthese, 136(1):31-56.

Heisenberg, W. (1973). Development of Concepts in the History of Quantum Theory. In Mehra, J., editor, The Physicist's Conception of Nature, pages 264-75. Dordrecht: Springer.

Heisenberg, W. (1976). The nature of elementary particles. Physics Today, 29(3):32-9.

Kantorovich, A. (2003). The priority of internal symmetries in particle physics. Studies in History and Philosophy of Science Part B: Studies in History and Philosophy of Modern Physics, 34(4):651-75.

Klein, F. (1893). Vergleichende Betrachtungen über neuere geometrische Forschungen. Mathematische Annalen, 43(1):63-100. Revised version of the booklet by the same title published by A. Duchert, Erlangen, in 1872.

Klein, F. (1910). Über die geometrischen Grundlagen der Lorentz-Gruppe. Jahresbericht der Deutschen Mathematiker-Vereinigung, 19:281-300. https://eudml.org/doc/ 145243, accessed 22 April 2020.

Kochen, S. (1996). Construction of Quantum Mechanics Via Commutative Operations. In Clifton, R., editor, Perspectives on Quantum Reality, volume 57 of The University of Western Ontario Series in the Philosophy of Science, pages 237-43. Dordrecht: Springer.

Ladyman, J. (1998). What is structural realism? Studies in the History and Philosophy of Science Part A, 29(3):409-24.

Ladyman, J., Ross, D., Spurrett, D., and Collier, J. (2007). Every Thing Must Go: Metaphysics Naturalized. Oxford: Oxford University Press.

Maudlin, T. (2018). Ontological Clarity via Canonical Presentation: Electromagnetism and the Aharonov-Bohm Effect. Entropy, 20(6). 465.

McKenzie, K. (2014). On the Fundamentality of Symmetries. Philosophy of Science, 81(5):1090-102.

McKenzie, K. (2017). Ontic Structural Realism. Philosophy Compass, 12(4).

Moretti, V. (2013). Spectral Theory and Quantum Mechanics: With an Introduction to the Algebraic Formulation. Milan: Springer. 
Nambu, Y. (1973). Generalized Hamiltonian Dynamics. Phys. Rev. D, 7:2405-412.

Ne'eman, Y. and Sternberg, S. (1991). Internal Supersymmetry and Superconnections. In Donato, P., Duval, C., Elhadad, J., and Tuynman, G., editors, Symplectic Geometry and Mathematical Physics, pages 326-54. Boston: Birkhäuser.

North, J. (2009). The "Structure" of Physics: A Case Study. The Journal of Philosophy, 106(2):57-88.

Roberts, B. W. (2011). Group Structural Realism. The British Journal for the Philosophy of Science, 62(1):47-69.

Rovelli, C. (2011). Zakopane lectures on loop gravity. arXiv:1102.3660. https://arxiv. org/abs/1102.3660, accessed 22 April 2020.

Schroeren, D. (2020). Symmetry Fundamentalism in Quantum Mechanics. manuscript.

Strocchi, F. (2005). Symmetry Breaking. Berlin: Springer.

Wallace, D. (2019). Who's afraid of coordinate systems? An essay on representation of spacetime structure. Studies in the History and Philosophy of Science Part B: Studies in History and Philosophy of Modern Physics, 67:125-36.

Weinberg, S. (1987). Towards the Final Laws of Physics. In Mackenzie, R. and Durst, P., editors, Elementary Particles and the Laws of Physics - The 1986 Dirac Memorial Lectures, pages 61-110. Cambridge: Cambridge University Press.

Weinberg, S. (1992). Dreams of a Final Theory. New York: Pantheon Books.

Weinberg, S. (1995). The Quantum Theory of Fields Vol. I. Cambridge: Cambridge University Press.

Wigner, E. P. (1931). Gruppentheorie und ihre Anwendung auf die Quantenmechanik der Atomspektren. Braunschweig: Vieweg.

Wigner, E. P. (1939). On Unitary Representations of the Inhomogeneous Lorentz Group. Annals of Mathematics, 40:149-204.

Wigner, E. P. (1968). Symmetry principles in old and new physics. Bulletin of the American Mathematical Society, 74(5):793-815.

Williamson, T. (2016). Modal science. Canadian Journal of Philosophy, 46(4-5):453-92.

Wolff, J. (2011). Do Objects Depend on Structures? The British Journal for the Philosophy of Science, 63(3):607-25. 